\title{
ON REAL ALGEBRAIC MODELS OF SMOOTH MANIFOLDS
}

\author{
J. BOCHNAK AND W. KUCHARZ
}

An affine nonsingular real algebraic variety $X$ diffeomorphic to a smooth manifold $M$ is said to be an algebraic model of $M$. The remarkable theorem of Nash-Tognoli asserts that each compact smooth manifold $M$ has an algebraic model [16 or 6, Theorem 14.1.10]. In fact, there exists an infinite family $\left\{X_{i}\right\}_{i \in \mathrm{N}}$ of irreducible algebraic models of $M$ such that $X_{i}$ and $X_{j}$ are birationally nonisomorphic for $i \neq j$ [10] (cf. also [7] for a proof in a special case). In view of these results, it seems natural and interesting to investigate algebro-geometric properties of various algebraic models of a given smooth manifold. This paper addresses a few questions of this type. For notions and results of real algebraic geometry we refer the reader to the book [6].

Given a compact affine nonsingular real algebraic variety $X$, denote by $H_{k}^{\text {alg }}(X, \mathbf{Z} / 2)$ the subgroup of $H_{k}(X, \mathbf{Z} / 2)$ of the homology classes represented by (Zariski closed) $k$-dimensional algebraic subvarieties of $X$ [6, Chapter 11 or 11]. Let $H_{\mathrm{alg}}^{k}(X, \mathbf{Z} / 2)$ be the image of $H_{d-k}^{\text {alg }}(X, \mathbf{Z} / 2)$, $d=\operatorname{dim} X$, under the Poincaré duality isomorphism $H_{d-k}(X, \mathbf{Z} / 2) \rightarrow$ $H^{k}(X, \mathrm{Z} / 2)$. Although the groups $H_{\mathrm{alg}}^{k}(X, \mathrm{Z} / 2)$ are one of the most important invariants of $X$ (a sample of applications can be found in $[1,2,3$, $6,8,9]$ ), our knowledge of their behavior is still rather limited. Here we consider the following.

Problem. Let $M$ be a compact smooth manifold and let $G$ be a subgroup of $H^{k}(M, Z / 2)$. When are there an algebraic model $X$ of $M$ and a diffeomorphism $\varphi: X \rightarrow M$ such that the induced isomorphism

$$
\varphi^{*}: H^{k}(M, \mathbf{Z} / 2) \rightarrow H^{k}(X, \mathbf{Z} / 2)
$$

maps $G$ onto $H_{\mathrm{alg}}^{k}(X, \mathbf{Z} / 2)$ ?

This problem has attracted the attention of several mathematicians (cf. $[3,4,5,6,12,14,15])$, however, the results are far from complete. We have a solution for $k=1, M$ connected, and $\operatorname{dim} M \geq 3$.

THEOREM 1. Let $M$ be a compact connected smooth manifold with $\operatorname{dim} M$ $\geq 3$ and let $G$ be a subgroup of $H^{1}(M, Z / 2)$. Then the following conditions are equivalent:

(i) There exists an algebraic model $X$ of $M$ and a diffeomorphism $\varphi: X \rightarrow$ $M$ such that $\varphi^{*}(G)=H_{\mathrm{alg}}^{1}(X, \mathbf{Z} / 2)$.

(ii) The first Stiefel-Whitney class $w_{1}(M)$ of $M$ is in $G$.

Received by the editors April 7, 1988.

1980 Mathematics Subject Classification (1985 Revision). Primary 57R19, 14G30, 14 C99.

The second author was supported by NSF Grant DMS-8602672 
In particular, if $M$ is orientable, then (i) is always satisfied.

For smooth surfaces and $k=1$ we have only a partial, but quite satisfactory, solution. First let us define the following invariants of a compact nonsingular real algebraic surface $X$ :

$$
\begin{gathered}
\beta(X)=\operatorname{dim}_{\mathbf{Z} / 2} H_{\mathrm{alg}}^{1}(X, \mathbf{Z} / 2), \\
\delta(X)=\operatorname{dim}_{\mathbf{Z} / 2}\left\{v \in H_{\mathrm{alg}}^{1}(X, \mathbf{Z} / 2) \mid v \cup v=0\right\} .
\end{gathered}
$$

If $X$ is connected, orientable (resp. nonorientable of odd topological genus), then $\beta(X)=\delta(X)$ (resp. $\beta(X)=\delta(X)+1$; indeed, $w_{1}(X)$ is in $H_{\mathrm{alg}}^{1}(X, \mathbf{Z} / 2)$ [6, Theorem 12.4.8] and $\left.w_{1}(X) \cup w_{1}(X) \neq 0\right)$. For $X$ connected, nonorientable of even topological genus, one has either $\beta(X)=\delta(X)$ or $\beta(X)=$ $\delta(X)+1$ and, in accordance with Theorem 2 below, all topologically possible cases can be realized algebraically.

THEOREM 2. Let $M$ be a compact connected smooth surface of genus $g$.

(i) If $M$ is orientable (resp. nonorientable of odd genus) and $\beta$ is an integer satisfying $0 \leq \beta \leq 2 g$ (resp. $1 \leq \beta \leq g$ ), then there exists an algebraic model $X_{\beta}$ of $M$ with $\beta\left(X_{\beta}\right)=\beta$.

(ii) If $M$ is nonorientable of even genus and $\beta$ and $\delta$ are integers satisfying either $\beta=\delta, 1 \leq \beta \leq g-1$, or $\beta=\delta+1,2 \leq \beta \leq g$, then there exists an algebraic model $X_{\beta, \delta}$ of $M$ such that $\beta\left(X_{\beta, \delta}\right)=\beta$ and $\delta\left(X_{\beta, \delta}\right)=\delta$.

Our interest in the invariants $\beta(X)$ and $\delta(X)$ is explained by the fact that they determine the projective module group $K_{0}(R(X))$ of the ring $R(X)$ of regular functions from $X$ to $\mathbf{R}$.

THEOREM 3. (i) Let $X$ be a compact connected affine nonsingular real algebraic surface. Then

$$
K_{0}(R(X)) \cong \mathbf{Z} \oplus(\mathbf{Z} / 4)^{\beta(X)-\delta(X)} \oplus(\mathbf{Z} / 2)^{\beta(X)+1-2(\beta(X)-\delta(X))} .
$$

(ii) As $X$ runs through all algebraic models of a compact, connected surface $M$ of genus $g$, then the groups $K_{0}(R(X))$ take, up to isomorphism, precisely $q(M)$ values, where

$$
q(M)= \begin{cases}2 g+1 & \text { if } M \text { is orientable, } \\ g & \text { if } M \text { is nonorientable and } g \text { is odd, } \\ 2 g-2 & \text { if } M \text { is nonorientable and } g \text { is even. }\end{cases}
$$

Condition (i) is proved in [9], while (ii) follows immediately from (i) and Theorem 2.

Here is another application. Given a compact affine nonsingular real algebraic variety $X$, let $C^{\infty}\left(X, S^{1}\right)$ denote the topological group of $C^{\infty}$ mappings from $X$ to the unit circle $S^{1}=\{z \in \mathrm{C}|| z \mid=1\}$ (the group structure on $C^{\infty}\left(X, S^{1}\right)$ is induced from that on $S^{1}$ and the topology is the $C^{\infty}$ one). Let $\overline{\mathcal{R}\left(X, S^{1}\right)}$ be the closure in $C^{\infty}\left(X, S^{1}\right)$ of the subgroup $R\left(X, S^{1}\right)$ of regular mappings from $X$ to $S^{1}$. Below we are concerned with the quotient group

$$
\Gamma(X)=C^{\infty}\left(X, S^{1}\right) / \overline{\mathcal{R}\left(X, S^{1}\right)} .
$$


THEOREM 4. Let $M$ be a compact connected smooth manifold with $\operatorname{dim} M$

$\geq 2$. Let

$$
r_{M}: H^{1}(M, \mathbf{Z}) \otimes \mathbf{Z} / 2 \rightarrow H^{1}(M, \mathbf{Z} / 2)
$$

be the canonical monomorphism and let

$$
\alpha(M)= \begin{cases}\operatorname{rank} H^{1}(M, Z)-1 & \text { if } M \text { is nonorientable, } \\
& \begin{array}{l}
\text { and } w_{1}(M) \in \text { Image } r_{M}, \\
\operatorname{rank} H^{1}(M, Z)
\end{array}\end{cases}
$$

(i) For each algebraic model $X$ of $M$, one has $\Gamma(X) \cong(\mathbf{Z} / 2)^{s}$ for some $s$ with $0 \leq s \leq \alpha(M)$.

(ii) For each integer $s$ satisfying $0 \leq s \leq \alpha(M)$, there exists an algebraic model $X_{s}$ of $M$ with $\Gamma\left(X_{s}\right) \cong(\mathbf{Z} / 2)^{s}$.

SKETCH OF PROOF. Let $X$ be a compact affine nonsingular real algebraic variety. By [8, Theorem 1.4], a mapping $f$ in $C^{\infty}\left(X, S^{1}\right)$ is in $\overline{\mathcal{R}\left(X, S^{1}\right)}$ if and only if $f^{*}\left(H^{1}\left(S^{1}, \mathbf{Z} / 2\right)\right)$ is contained in $H_{\text {alg }}^{1}(X, \mathbf{Z} / 2)$. It follows that $\Gamma(X)$ is isomorphic to the quotient group $A / B$, where $A=$ Image $r_{X}$ and $B=A \cap H_{\text {alg }}^{1}(X, \mathrm{Z} / 2)$. This implies (i) and, applying Theorems 1 and 2, also (ii).

Proofs of Theorems 1 and 2 are quite involved. Here we can only sketch the proof of Theorem 1.

The implication (i) $\Rightarrow$ (ii) is well known (cf. [6, Theorem 12.4.8]). Suppose then that (ii) holds. One easily finds a $C^{\infty}$ embedding $i: M \rightarrow P$ of $M$ into the product $P=\mathbf{R} P^{k_{1}} \times \cdots \times \mathbf{R} P^{k_{r}}$ of real projective spaces with $i^{*}\left(H^{1}(P, \mathbf{Z} / 2)\right)=G$. It requires some care to construct a compact smooth surface $S$ in $M$ such that $j^{*}(v) \neq 0$ for all $v$ in $H=H^{1}(M, \mathbf{Z} / 2) \backslash G$, where $j: S \rightarrow M$ is the inclusion mapping, and $S$ bounds a compact smooth submanifold $W$ of $M$ with the property that the normal vector bundles of $i(W)$ in $i(M)$ and $P$ are trivial. Using the triviality of the normal vector bundle of $i(W)$ in $P$, one shows the existence of a $C^{\infty}$ diffeomorphism $h: P \rightarrow P$, arbitrarily close in the $C^{\infty}$ topology to the identity mapping, such that $Y=h(i(S))$ is a nonsingular algebraic subvariety of $P$ (cf. [12, $\S 2])$. Moreover, and this is the hard part of the construction, $h$ can be chosen in such a way that $H_{\text {alg }}^{1}(Y, Z / 2)=0$. To achieve this, one uses, in particular, appropriate real algebraic versions of the theorem of Gherardelli [13, Theorem 6.5] and the theorem of Noether-Lefschetz-Moishezon [13, Theorem 7.5] concerning the Picard group of complex projective varieties.

Since $H_{\mathrm{alg}}^{*}(P, \mathrm{Z} / 2)=H^{*}(P, \mathrm{Z} / 2)$ and $Y$ has trivial normal vector bundle in $N=h(i(M))$, it follows from [1, Proposition 2.8] that there exist a positive integer $q$ and a $C^{\infty}$ embedding $e: N \times\{0\} \rightarrow P \times \mathbf{R}^{q}$, arbitrarily close in the $C^{\infty}$ topology to the inclusion mapping $N \times\{0\} \rightarrow P \times \mathbf{R}^{q}$, such that $X=e(N \times\{0\})$ is a nonsingular algebraic subvariety of $P \times$ $\mathbf{R}^{q}$ containing $Y \times\{0\}$. Clearly, $\varphi: X \rightarrow M$, defined by the condition $e(h(i(\varphi(x))), 0)=x$ for $x$ in $X$, is a $C^{\infty}$ diffeomorphism and $H_{\mathrm{alg}}^{1}(X, \mathbf{Z} / 2)$ contains $\varphi^{*}(G)$. Since $H_{\text {alg }}^{1}(Y \times\{0\}, \mathbf{Z} / 2)=0$ and $f^{*}\left(\varphi^{*}(v)\right) \neq 0$ for all 
$v$ in $H$, where $f: Y \times\{0\} \rightarrow X$ is the inclusion mapping, it follows that $\varphi^{*}(G)=H_{\text {alg }}^{1}(X, Z / 2)$, i.e., (i) is satisfied.

\section{REFERENCES}

1. S. Akbulut and H. King, The topology of real algebraic sets with isolated singularities, Ann. of Math. (2) 113 (1981), 425-446.

2. __ A relative Nash theorem, Trans. Amer. Math. Soc. 267 (1981), 465-481.

3. __ Submanifolds and homology of nonsingular real algebraic varieties, Amer. J. Math. 107 (1985), 45-83.

4. R. Benedetti and M. Dedò, Counterexamples to representing homology classes by real algebraic subvarieties up to homeomorphism, Compositio Math. 53 (1984), 143-151.

5. R. Benedetti and A. Tognoli, Remarks and counterexamples in the theory of real algebraic vector bundles and cycles, Géométrie Algébrique Réelle et Formes Quadratiques, Lecture Notes in Math., vol. 959, Springer-Verlag, Berlin and New York, 1982, pp. 198-211.

6. J. Bochnak, M. Coste and M.-F. Roy, Géométrie algébrique réelle, Ergeb. Math. Grenzgeb., vol. 12, Springer-Verlag, Berlin and New York, 1987.

7. J. Bochnak and W. Kucharz, Nonisomorphic algebraic structures on smooth manifolds, Proc. Amer. Math. Soc. 101 (1987), 424-426.

8. __, Algebraic approximation of mappings into spheres, Michigan Math. J. 34 (1987), 119-125.

9. __ K-theory of real algebraic surfaces and threefolds (to appear).

10. _ Algebraic models of smooth manifolds (a preliminary version), preprint, Univ. of New Mexico, 1988, pp. 45.

11. A. Borel and A. Haefliger, La classe d'homologie fondamentale d'un espace analytique, Bull. Soc. Math. France 89 (1961), 461-513.

12. W. Kucharz, On homology of real algebraic sets, Invent. Math. 82 (1985), 19-26.

13. B. G. Moishezon, Algebraic homology classes on algebraic varieties, Math. USSR Izv. 1 (1967), 209-251.

14. J.-J. Risler, Sur l'homologie des surfaces algébriques réelles, Géométrie Algébrique Réelle et Formes Quadratiques, Lecture Notes in Math., vol. 959, Springer-Verlag, Berlin and New York, 1982, pp. 381-385.

15. R. Silhol, $A$ bound on the order of $H_{n-1}^{(a)}(X, Z / 2)$ on a real algebraic variety, Géométrie Algébrique Réelle et Formes Quadratiques, Lecture Notes in Math., vol. 959, Springer-Verlag, Berlin and New York, 1982, pp. 443-450.

16. A. Tognoli, Su una congettura di Nash, Ann. Scuola Norm. Sup. Pisa 27 (1973), 167185.

\footnotetext{
Department of Mathematics, Vrije Universiteit, 1007 MC Amsterdam, The NETHERLANDS
}

Department of Mathematics and Statistics, University of New Mexico, AlbuQUERQUe, NeW MEXICO 87131 